\title{
Neighbourhood characteristics and under-five mortality in Nigeria
}

\author{
Sunday A. Adedini ${ }^{1,2}$ \\ ${ }^{1}$ Demography and Population Studies Programme, Schools of Public Health and Social Sciences, \\ University of the Witwatersrand, Johannesburg, South Africa \\ ${ }^{2}$ Demography and Social Statistics Department, Faculty of Social Sciences, Obafemi Awolowo \\ University, Ile-Ife, Nigeria \\ Email: sadedini@cartafrica.org
}

\begin{abstract}
Despite global decline in childhood mortality, under-5 mortality remains high in Nigeria. While many studies have reported individual level factors as important determinants of under-five mortality in Nigeria, similar studies on the effects of neighbourhood contexts have been minimal. Hence, this study examines the effects of neighbourhood contexts on under-5 mortality in Nigeria. Using 2003 and 2008 Nigeria Demographic and Health Survey (NDHS) data, multilevel Cox regression analysis was performed on a nationally representative sample of 6,028 children (2003 NDHS) and 28,647 children (2008 NDHS). Results indicated neighbourhood context as important factor for child survival. For instance, findings showed that being born or raised in poor neighbourhoods (HR:I.54,p<0.05), rural communities (HR:I.25,p<0.05), and North-eastern region of Nigeria (HR: I.56,p<0.05) was associated with elevated hazards of death before age 5. Findings of this study suggest that achieving improved neighbourhood contexts holds great potentials for acceleration of under-five mortality reduction in Nigeria.
\end{abstract}

Keywords: Under-five mortality, child survival, neighbourhood, community, Nigeria

\section{Résumé}

Malgré baisse globale de la mortalité infantile, moins de 5 ans reste élevé au Nigeria. Alors que de nombreuses études ont rapporté des facteurs individuels comme des déterminants importants de mortalité des moins de cinq ans au Nigeria, des études similaires sur les effets des contextes et des quartiers ont été minimes. Ainsi, cette étude examine les effets de contextes de quartier sur sous 5 mortalité au Nigeria. Utilisation de 2003 et l'Enquête Démographique et de Santé 2008 Nigeria (NDHS), l'analyse de régression de Cox à plusieurs niveaux a été réalisée sur un échantillon national représentatif de 6028 enfants ( 2003 NDHS) et 28647 enfants (2008 NDHS) . Les résultats ont indiqué contexte du quartier comme un facteur important pour la survie de l'enfant. Par exemple, les résultats ont montré que d'être né ou a grandi dans les quartiers pauvres (HR: I,54, $p<0,05)$, les communautés rurales $(H R: I, 25, p<0,05)$, et la région NordEst du Nigeria (HR: I,56, $p<0,05)$ a été associée à des risques élevés de décès avant 5 ans. Les résultats de cette étude suggèrent que la réalisation de l'amélioration des contextes de quartier détient un grand potentiel pour l'accélération de la réduction de la mortalité des moins de cinq ans au Nigeria.

Mots-clés: moins de cinq ans, la survie de l'enfant, le quartier, la communauté, Nigeria

\section{Introduction}

Despite the global decline in childhood mortality, demographic and public health literature indicates that child health outcomes are generally poor in the sub-Saharan Africa. In 20II, the variation in underfive mortality rates between the developing and developed nations is more than 78-fold ranging from 180 per 1000 live births in Angola to only 2.31 per 1000 live births in Singapore (World Factbook, 20II). Worse still, evidence showed that only about one-third of all countries in Africa showed a decline of $30 \%$ or more in under-five mortality, while many countries sadly showed a considerable increase in the recent time (Becher, 20I0). In Nigeria, with about $I$ in 6 children dying before the age of five
(NPC \& ICF Macro, 2009), the country like many other nations in sub-Saharan Africa, is not on track to achieve the Millennium Development Goal 4 (reducing childhood mortality) by 2015 (Lykens et al., 2009).

Nigeria remains a major contributor to global statistics on under-five mortality. The country's rate of under-five mortality - 156.9 per 1000 live births (ICF Macro and NPC, 2009) - is among the highest in the world. Besides, there is a substantial geographic variation in the levels of under-five mortality in the country - from 89 per 1000 live births in the South-west to 222 deaths per 1000 live birth in the North-east.

To understand the factors driving high under-five 
mortality in Nigeria and other parts of sub-Saharan Africa, many studies with diverse findings have been reported. While many studies on childhood mortality in Nigeria have indicated that individual level factors such as maternal education and other socioeconomic factors are important predictors of underfive mortality (Adebowale et al., 2012; Fayeun \& Omololu, 201 I; Grais et al., 2007; Nwokocha \& Awomoyi, 2009), studies on the effects of neighbourhood contexts on child survival have been few. Meanwhile, literature has established that living in a deprived neighbourhood or community is associated with poor health outcomes of individuals (Harttgen \& Misselhorn, 2006; Omariba et al., 2007; Sastry, 1997; Zanini et al., 2009). For instance, Zanini et al (2009) found that about half of the variability in infant mortality rates in Brazil was due largely to community-level characteristics. Sastry (1997) argued that neighbourhood characteristics can aggravate or alleviate mortality risks of individuals depending on the neighbourhood where individuals reside.

Recent evidences have continued to underscore the importance of neighbourhood characteristics on health outcomes. Aminzadeh et al (2013) found an association between neighbourhood deprivation and wellbeing of young people. Unger (2013) observed that areas of broad economic and social disadvantage (due to overcrowding, substandard housing, poor water and sanitation) tend to have higher under-five mortality compared to socially and economically advantaged areas. Although a study by Gilbert et al (2013) established a decline in infant mortality across neighbourhood income quintiles over time in Canada, the study indicates the need for infant health promotion policies among the vulnerable populations. A study by Becares et al (2013) established association between area deprivation and poor health outcomes, and concludes that addressing neighbourhood poverty and area deprivation is essential to improving health outcomes of individuals.

Although, the effect of neighbourhood contexts on child survival has been recognised in a number of countries in sub-Saharan Africa (Antai, 20l l b; Boco, 2010; Omariba, et al., 2007) and elsewhere (Sastry, 1997), evidence is sparse on the effects of neighbourhood contexts on under-five mortality in Nigeria.

Several studies on under-five mortality in Nigeria seem to emphasize individual-level determinants and clinical-related causes of infant and child deaths, e.g.; (Ekenze et al., 2009; Ifesanya et al., 2009; Melliez et al., 2007; Okafor et al., 2009; Okoro et al., 2009). Many other studies that are not hospital-based, but population-based failed to consider the influence of contextual situation where children are raised on their survival chances. To this end, the present study seeks to advance the existing knowledge beyond the understanding of clinical-related or individual-level factors by examining the influences of broad familial and neighbourhood contexts on under-five mortality in Nigeria.

\section{Theoretical framework}

This study has its theoretical underpinning in two theoretical models - (I) Mosley-Chen model and (2) Sastry Framework. Mosley and Chen in 1984 postulated a model which proposes that socio-economic determinants of child mortality operate through a common set of biological and proximate mechanisms to influence child mortality (WHO, 2003). The model takes into account a range of factors grouped into three broad categories: individuallevel variables (i.e. individual productivity - at mothers or fathers' level as well as traditions/ norms/attitudes); household-level variables (e.g. income and wealth) and community-level variables (i.e. ecological setting; political economy and health system). Building upon the premise established by Mosley-Chen model, Sastry (1997) categorized the proximate determinants into three broad categories: genetic, behavioural and environmental. He argued that these determinants can occur at three different levels of operation: child, family and community; and that the three levels provide a logical organization for the variables that are likely to influence child mortality.

The thrust of the two models is that children belonging to the same household are exposed to the same situation while children in the same neighbourhood are exposed to the same neighbourhood situation. Thus in this paper, the relationship between neighbourhood context and child survival was conceptualized based on the foundation established by these two theoretical models.

\section{Data and method}

This study draws on 2003 and 2008 Nigeria Demographic and Health Survey (NDHS) data. Data from 2003 and 2008 NDHS were utilized in this study purposely to understand the influence of neighborhood contexts on child survival within a ten-year period - 1998-2008. The two surveys adopted similar methodology and this makes results comparable. The primary sampling unit (PSU) which was regarded as a cluster for both surveys was defined on the basis of Enumeration Areas (EAs). While the 2003 NDHS adopted the Enumeration Areas designed for 1991 population census, the 2008 NDHS utilized the Enumeration Areas designed for 
Nigeria 2006 population and housing census. Samples for the two surveys were selected using stratified two-stage cluster design consisting of 365 clusters for 2003 NDHS (NPC and ORC Macro, 2004) and 888 clusters for 2008 NDHS (NPC and ICF Macro, 2009). Data were gathered from 7620 women aged 15-49 in 2003 and 33,385 women aged 15-49 women in 2008. The relevant data for this study (women age 15-49 years who had at least one live birth within the five years preceding the survey) were extracted from the whole 2003 and 2008 NDHS datasets.

Out of the survey's complete sample size of 7620 women contained in 2003 dataset, the sample size for this paper comprised 3775 women who had a total of 6028 live births within the five years before the survey. Similarly, from a total of 33,385 women contained in the 2008 dataset, the sample size for this study comprised 18,028 women who had a total of 28,647 live births within the five years preceding the survey. The birth recode datasets of both surveys were utilized in the analysis. The data contained information for each live birth with maternal variables replicated for births to the same mother.

To account for oversampling of some sections of the population, weighting factor provided by Measure DHS was applied in data management and analysis. Data quality assessment indicates that both datasets were of good quality. For instance, data quality assessment test for 2008 NDHS data indicates that the percentage of missing information on births and deaths only varied between around I\% and 3\% (NPC \& ICF Macro, 2009).

\section{Variables measurement}

\section{Outcome variable}

The outcome variable for this study is the risk of death during the first five years of life. This is defined as the risk of a child dying between birth and the fifth birthday. The variable was measured as the duration of survival since birth in months.

\section{Independent variables}

The independent variables in this paper included such important characteristics at the individual level, familial level (household level) and community level. The selection of independent variables in this study was guided by the reviewed literature and the theoretical foundation established from the literature.

The variables at the individual level included characteristics at the child and mother levels. These are maternal age, child's sex, birth order, birth interval, child's size at birth (the self-reported size of the child at birth), prenatal care, contraceptive use, place of delivery and maternal education. Important selected household-level variables which could influence child survival include: family structure, children ever born and wealth index. The selected community-level variables in this study are: region of residence, place of residence, ethnic diversity, distance to health facility, community maternal level of education, community infrastructures, community prenatal care, community poverty level and community hospital delivery. Apart from region of residence, place of residence and distance to health facility, all other community level characteristics were created from the individual-level and household-level variables. Using Stata software (version II.I), individual and household-level variables were aggregated at the level of PSU to create the community-level variables of interest. The generated community-level variables were divided into three tertiles and then categorized as low, medium, and high. The decision to create the community variables considered in this study was based on the understanding obtained from the reviewed literature.

\section{Data analysis}

Descriptive and inferential statistics were employed in data analysis. The background characteristics of the study sample were presented using percentage distribution. Multilevel Cox proportional hazards model was employed to examine association between the outcome and the explanatory variables; and to examine measures of variation in under-five mortality across contexts. All analysis was done using Stata (version II.I). The multilevel Cox regression analysis was performed using the generalized linear latent and mixed models (GLLAMM) procedure downloadable and implementable in Stata (RabeHesketh et al., 2004).

\section{Multilevel Cox proportional hazards model: methodological procedure}

A number of researchers had employed multilevel analysis to identify correlates of under-five mortality. The assumption is that "individuals (level I) are nested within households (level 2), and households are nested within communities (level 3)", (Harttgen and Misselhorn, 2006:6). To date, with the exception of one or two studies, Nigerian studies on under-five mortality have rarely employed multilevel model approach. The multilevel approach is good at identifying the broad social, economic and environmental contexts in which a child lives and experiences a particular health outcome (Griffiths et al., 2004). Individuals with similar household characteristics can have different health outcomes when residing in different communities with contrasting characteristics. Griffiths and colleagues (2004) opined that it would 
be methodologically wrong to fit a single-level standard regression model in the analysis of child survival. This is because standard regression models cannot handle hierarchical structure in the datasets due to its assumption of independence. In this paper, therefore, multilevel Cox proportional hazards regression analysis was undertaken to determine the extent to which the contextual determinants explain underfive mortality in Nigeria.

Further, Cox proportional hazards model (i.e. survival analysis) is appropriate in analysing censored observations. This means that, using Cox proportional hazards regression analysis, both the children's survival status and the time when the child died (or censored) were combined to generate the outcome variable. The Cox regression procedure is a useful technique for analysis of survival data and it takes care of censoring problem in mortality data. This is because some children are not fully exposed to the mortality risk. In social science research, an observation is said to be censored when the outcome of interest has not occurred. Cox regression analysis allows for the inclusion of censored observation and it models censored time-until-event data as a dependent variable where it can be assumed that the covariates have a multiplying effect on the baseline hazard.

The outcome variable in this study was treated as the time between birth and death of a child under age five years; or until the observation is censored. Children known to have died (i.e. non-censored) were regarded as the cases, while children who were still alive at the time of the survey were treated as right-censored observations. The probability of under-five mortality is called the hazard. The hazard was modelled using the following equation:

$h_{i}(\mathrm{t})=\mathrm{h}_{0}(\mathrm{t}) \times \exp \left(\mathrm{b}_{1} \mathrm{x}_{1}+\mathrm{b}_{2} \mathrm{x}_{2}+\cdots+\mathrm{b}_{\mathrm{k}} \mathrm{x}_{\mathrm{k}}\right)$

Where $\mathrm{x}_{1} \mathrm{x}_{1} \ldots \mathrm{x}_{\mathrm{k}}$ are a collection of explanatory variables and $\mathrm{H}_{0}(\mathrm{t})$ is the baseline hazard at time $\mathrm{t}$, representing the hazard for a person with the value 0 for all the explanatory variables (Fox, 2002). By dividing both sides of equation I by $\mathrm{H}_{0}(\mathrm{t})$ and taking logarithms, the equation I becomes:

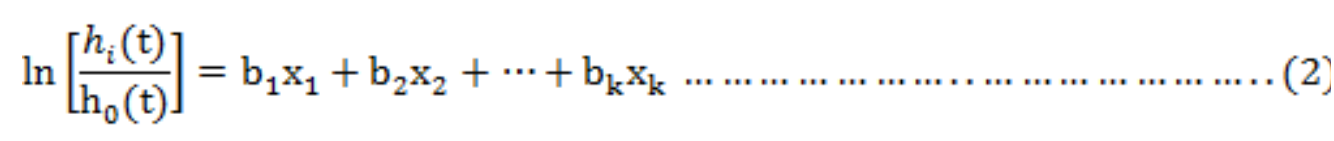

Where $\mathrm{H}(\mathrm{t}) / \mathrm{H}_{0}(t)$ is regarded as the hazard ratio. The coefficients $\mathrm{b}_{1} \ldots b_{k}$ are estimated by Cox regression. To estimate both the fixed and random effects in the multilevel survival analysis, it could be assumed that the hazards of any two units are proportional (Rabe-Hesketh et al., 2004) and this is modelled as:

$h_{i j}(t)=h^{0}(t) \exp \left(v_{i j}\right)$

In the above equation, there are two levels - (i.e. the two subscripts) - where $i$ represents the level l units and $j$ stands for the level two units, $v_{i j}$ denotes the linear predictor of GLLAMM.

Further, the multilevel analysis involved fitting seven models each using 2003 and 2008 DHS datasets. These models provide understanding on the influences of contextual factors on children's survival chances. Model 0 is the empty or null model and contains no explanatory variables, but focused mainly on decomposing total variance into both individual and community levels components. Model I considered only the region of residence in order to examine the independent influence of region where children were born or raised on their survival chances. Model 2 incorporated the child-level variables into the multilevel analysis. Model 3 incorporated the mother-level (familial) variables into the multilevel analysis. Model 4 considered only the contextual factors in order to examine the effect of community-level factors on child survival; independent of other factors. Model 5 is the full model that incorporated all variables into the multilevel analysis. Model 6 is the final model. Fitting this model 
involved two steps. First, stepwise survival analysis was done to determine the key variables associated with under-five mortality. Second, all the variables selected using stepwise Cox regression analysis were incorporated into the multilevel model.

Measures of association (i.e. fixed effects) were expressed in this paper as hazard ratios (HR) and $p$ value $(\alpha=0.05)$. The random effects which were regarded as measures of variations in under-five mortality across communities were expressed in this paper as intra-class correlation (ICC) (or variance partition coefficient (VPC), and proportional change in variance (PCV). The precision of random effects was determined by the standard error (SE) of the covariates. To determine the goodness of fit of the consecutive models, regression diagnostic was done using Akaike Information Criteria (AIC). Introduced by Hitrotugu Akaike in 197I, AIC measures the relative goodness of fit of statistical model (Reviews,
1988). Lower value of AIC indicates a better fit (Boco, 20I0).

\section{Results}

\section{Descriptive results}

Results presented in Table I revealed that proportion of the children was almost the same for males and females in 2003 (50.8\% vs. $49.2 \%)$ and 2008 (50.9\% vs. $49.1 \%)$. For both surveys, Table I also shows that percentage of children was highest for children of birth order 2-4, children with preceding birth interval of 2 years or more, children with large size at birth, children of uneducated women, children of women aged 25-34 years, children of currently married women, children delivered at home, children of Muslim mothers, children from poorest households and children whose mothers had never used contraceptive.

Table I Percentage distribution of study sample by background characteristics

\begin{tabular}{|c|c|c|c|c|}
\hline \multirow[t]{2}{*}{ Characteristics } & \multicolumn{2}{|l|}{2003} & \multicolumn{2}{|l|}{2008} \\
\hline & Frequency & Percentage & Frequency & Percentage \\
\hline \multicolumn{5}{|l|}{ Child's sex } \\
\hline Male & 3061 & 50.8 & 14604 & 50.9 \\
\hline Female & 2967 & 49.2 & 14043 & 49.1 \\
\hline \multicolumn{5}{|l|}{ Birth order } \\
\hline First births & 1200 & 19.9 & 5353 & 19.1 \\
\hline $2-4$ & 2621 & 43.5 & 13069 & 46.2 \\
\hline $5+$ & 2207 & 36.6 & 10225 & 34.7 \\
\hline \multicolumn{5}{|l|}{ Preceding birth interval } \\
\hline$<2$ years & 1124 & 18.7 & 5375 & 19.2 \\
\hline 2 years or more & 4904 & 81.3 & 23272 & 80.8 \\
\hline \multicolumn{5}{|l|}{ Birth size } \\
\hline Very large/larger than average & 2497 & 42.1 & 8449 & 47.4 \\
\hline Average & 2557 & 43.2 & 6685 & 38.1 \\
\hline Smaller than average/very small & 871 & 14.7 & 2702 & 14.5 \\
\hline \multicolumn{5}{|l|}{ Maternal education } \\
\hline None & 3032 & 50.3 & 14418 & 46.5 \\
\hline Primary & 1473 & 24.4 & 6552 & 23.2 \\
\hline Secondary & 1308 & 21.7 & 6338 & 24.9 \\
\hline Tertiary & 215 & 3.6 & 1339 & 5.4 \\
\hline \multicolumn{5}{|l|}{ Maternal current age } \\
\hline I5-24 years & 1610 & 26.7 & 7249 & 24.8 \\
\hline $25-34$ years & 3003 & 49.8 & $|4|||$ & 49.8 \\
\hline $35+$ & 1415 & 23.5 & 7287 & 25.4 \\
\hline \multicolumn{5}{|l|}{ Maternal age at child's birth } \\
\hline$<18$ & 503 & 8.3 & 2160 & 7.5 \\
\hline $15-24$ & 2167 & 36.0 & 9742 & 34.0 \\
\hline $25-34$ & 2456 & 40.7 & 12313 & 43.0 \\
\hline $35+$ & 902 & 15.0 & 4432 & 15.5 \\
\hline
\end{tabular}

Mother's marital status 


\begin{tabular}{|c|c|c|c|c|}
\hline Never married & 109 & 1.81 & 506 & 1.7 \\
\hline Currently married & 5707 & 94.7 & 27378 & 96.8 \\
\hline Previously married & 212 & 23.5 & 446 & 1.5 \\
\hline \multicolumn{5}{|l|}{ Received prenatal care } \\
\hline No & 1343 & 35.7 & 7015 & 36.8 \\
\hline Yes & 2420 & 64.3 & 10789 & 63.2 \\
\hline \multicolumn{5}{|l|}{ Place of delivery } \\
\hline Home & 3853 & 64.8 & 11794 & 63.9 \\
\hline Health facility & 2094 & 35.2 & 5908 & 36.1 \\
\hline \multicolumn{5}{|l|}{ Family structure } \\
\hline Monogamous & 3688 & 65.0 & 18063 & 67.9 \\
\hline Polygynous & 1989 & 35.0 & 9128 & 32.1 \\
\hline \multicolumn{5}{|l|}{ Religion } \\
\hline Catholic/Other Christians & 2307 & 38.3 & 11738 & 43.1 \\
\hline Muslim & 3598 & 59.7 & 16152 & 55.4 \\
\hline Others & 123 & 2.0 & 547 & 1.6 \\
\hline \multicolumn{5}{|l|}{ Wealth quintile } \\
\hline Poorest & 1405 & 23.3 & 7604 & 26.5 \\
\hline Poorer & $|32|$ & 21.9 & 6871 & 24.0 \\
\hline Average & 1183 & 19.6 & 5609 & 19.6 \\
\hline Richer & 1149 & 19.1 & 4755 & 16.6 \\
\hline Richest & 970 & 16.1 & 3808 & 13.3 \\
\hline \multicolumn{5}{|l|}{ Contraceptive Use } \\
\hline Never used & 5154 & 85.5 & 24952 & 84.8 \\
\hline Used modern method & 294 & 4.9 & 1072 & 4.9 \\
\hline Used traditional method & 580 & 9.6 & 2623 & 10.3 \\
\hline
\end{tabular}

\section{Random effects}

In this paper, random effects (i.e. measures of variation) represent the extent to which children raised in the same neighbourhood or community are exposed to the same neighbourhood situations such as availability (or non-availability) of health services, medical personnel, electricity, drinkable water, good sanitary conditions and others. From the multilevel modelling, the results presented in the null model which contains no explanatory variables, (Model 0 , Tables 2 and 3 ) indicated a significant variation in under-five mortality with variances 0.20 (2003) and 0.14 (2008) across individual level, and 0.25 (2003) and 0.21 (2008) across community level. The results from analysis of both surveys indicated that the between communities variance (expressed as VPC or ICC) in under-five mortality was slightly larger in 2003 (7.1\%) than in 2008 (6.0\%). Adding region of residence covariate into Model I; child-level variables into Model 3 and mother-level variables into Model 3 (Tables 2 and 3) also yielded significant variance across communities. For instance, the estimated
PCV in Model I indicated that $16.0 \%$ (2003) and $33.3 \%$ (2008) of the variances in under-five mortality across communities were explained by regions where children were born or raised.

Variances that resulted from inclusion of only community-level characteristics in the multilevel modelling were significant across communities (Model 4 Tables 2 and 3). ICC of 4.1\% (2003) and $2.7 \%$ (2008) were found for under-five death. Results from 2003 dataset showed that, the PCV of $44.0 \%$ (Model 4 Table 2) in the hazards of dying before age five, and PCV of $57.1 \%$ (Model 4 Table 3) in the risks of under-five death, could be attributed to community-level characteristics.

Results from both datasets indicated that PCV of 64.0\% (2003 NDHS) and 81.0\% (2008 NDHS) in the risks of under-five death were partly due to individual- and community-level factors included in the final model (Model 6); while unexplained variations of $36 \%$ (NDHS 2003) and 19\% (NDHS 2008) were due to unobserved/unmeasured factors. 
Table 2 Child and mother-level compositional and community contextual factors associated with regional variations in under-five mortality in Nigeria, 2003

\begin{tabular}{|c|c|c|c|c|c|c|c|}
\hline Characteristics & Model 0 & Model I & Model 2 & Model 3 & Model 4 & Model 5 & Model 6 \\
\hline Fixed effects & $\begin{array}{l}\text { Empty Model } \\
\text { (HR) }\end{array}$ & $\begin{array}{l}\text { Region } \\
\text { covariate } \\
(\mathbf{H R})\end{array}$ & $\begin{array}{l}\text { Child-level } \\
\text { variables } \\
\text { (HR) }\end{array}$ & $\begin{array}{l}\text { Mother-level } \\
\text { variables } \\
\text { (HR) }\end{array}$ & $\begin{array}{l}\text { Communit } \\
\text { y-level } \\
\text { variables } \\
(\mathrm{HR})\end{array}$ & $\begin{array}{l}\text { Full model } \\
\text { (HR) }\end{array}$ & $\begin{array}{l}\text { Final } \\
\text { Model } \\
(\text { HR) }\end{array}$ \\
\hline \multicolumn{8}{|l|}{ Region of residence } \\
\hline South-west & & I & I & I & I & 1 & I \\
\hline North-central & & $1.45 *$ & $1.52 *$ & 1.14 & 1.08 & 0.92 & 0.80 \\
\hline North-east & & $2.09 *$ & $2.00 *$ & 1.43 & 1.37 & 1.24 & 0.80 \\
\hline North-west & & $2.00 *$ & I.83* & 1.46 & 1.38 & 1.22 & 0.72 \\
\hline South-east & & $1.36 *$ & 0.95 & 0.66 & 1.37 & 0.74 & 0.68 \\
\hline South-south & & I.68* & I.78* & I.54* & 1.42 & 1.98 & 1.64 \\
\hline \multicolumn{8}{|l|}{ Child's sex } \\
\hline Male & & & I & & & 1 & \\
\hline Female & & & 0.94 & & & 0.95 & \\
\hline \multicolumn{8}{|l|}{ Birth order } \\
\hline First birth & & & I & & & 1 & I \\
\hline $2-4$ & & & 1.00 & & & 0.73 & $0.59 *$ \\
\hline $5+$ & & & $2.73^{*}$ & & & $2.30 *$ & 0.73 \\
\hline \multicolumn{8}{|l|}{ Birth interval } \\
\hline$<24$ months & & & 1 & & & 1 & 1 \\
\hline $24+$ & & & $0.68 *$ & & & 0.76 & 0.74 \\
\hline \multicolumn{8}{|l|}{ Child size at birth } \\
\hline Large & & & I & & & 1 & I \\
\hline Average & & & 1.03 & & & 1.03 & 1.04 \\
\hline Small & & & $1.38 *$ & & & $\mathrm{I} .47^{*}$ & $1.45^{*}$ \\
\hline \multicolumn{8}{|l|}{ Maternal education } \\
\hline None & & & & I & & 1 & I \\
\hline Primary & & & & 0.97 & & 1.36 & I.37* \\
\hline Secondary or higher & & & & $0.70 *$ & & 0.82 & 0.91 \\
\hline \multicolumn{8}{|l|}{ Mother's age } \\
\hline $15-24$ & & & & I & & 1 & I \\
\hline $25-34$ & & & & 0.83 & & 1.03 & 0.90 \\
\hline $35+$ & & & & 1.04 & & $2.00 *$ & 1.36 \\
\hline \multicolumn{8}{|l|}{ CEB } \\
\hline$<3$ & & & & I & & 1 & I \\
\hline $3-4$ & & & & $2.33^{*}$ & & $2.30 *$ & $1.96 *$ \\
\hline $5+$ & & & & $4.02 *$ & & $3.29 *$ & $3.10 *$ \\
\hline \multicolumn{8}{|l|}{ Family structure } \\
\hline Monogamous & & & & I & & 1 & I \\
\hline Polygynous & & & & $1.23 *$ & & 1.23 & 1.22 \\
\hline \multicolumn{8}{|l|}{ Religion } \\
\hline Christianity & & & & I & & 1 & \\
\hline Islam & & & & 1.10 & & 0.96 & \\
\hline Others & & & & 0.92 & & 0.78 & \\
\hline \multicolumn{8}{|l|}{ Wealth index } \\
\hline Poorest & & & & I & & 1 & \\
\hline
\end{tabular}




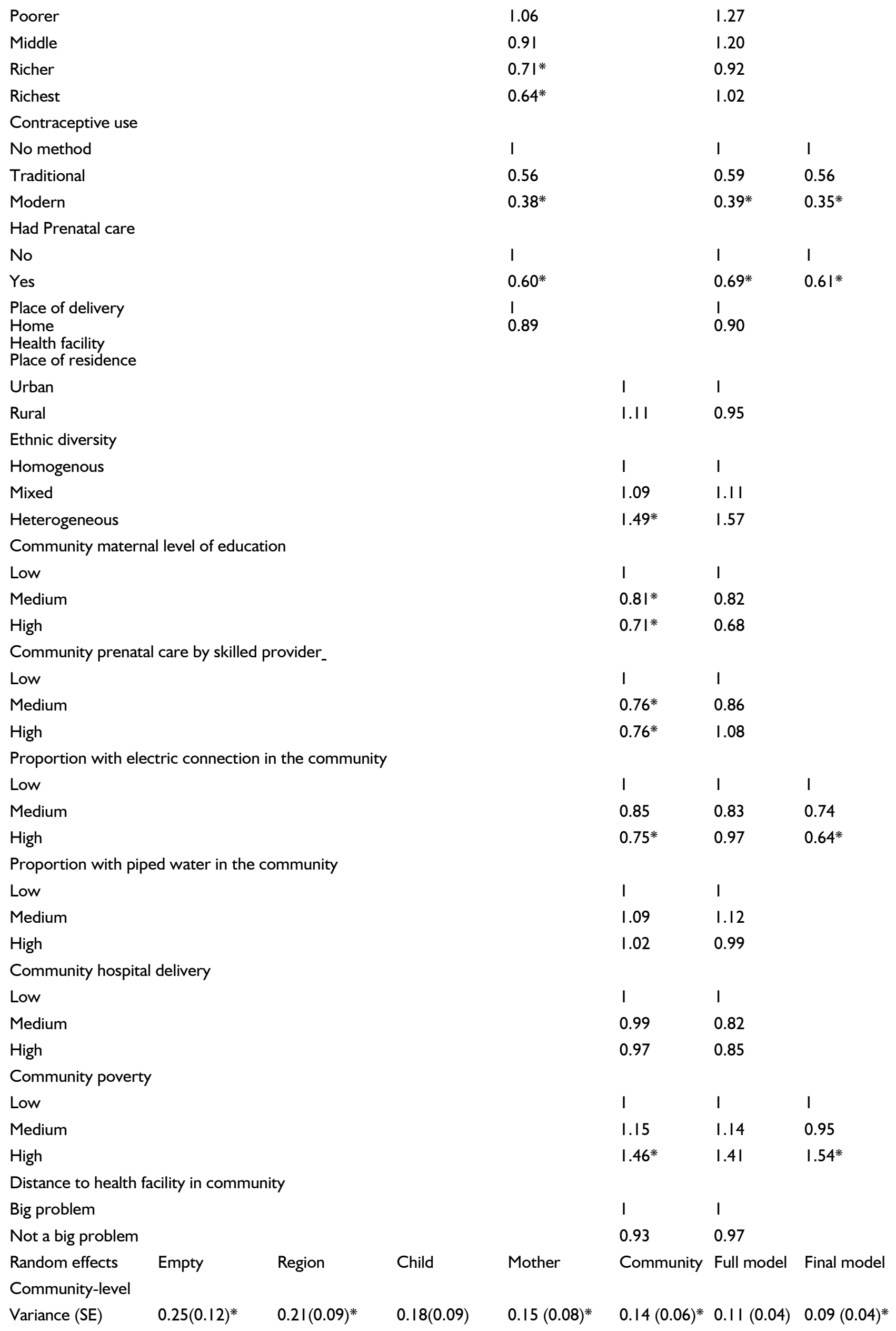




$\begin{array}{llllllll}\text { VPC/ICC (\%) } & 7.1 & 6.0 & 5.2 & 4.4 & 4.1 & 3.2 & 2.7 \\ \begin{array}{l}\text { Explained variation } \\ \text { (PCV) \% }\end{array} & \text { Reference } & 16.0 & 28.0 & 40.0 & 44.0 & 56.0 & 64.0 \\ \begin{array}{l}\text { Child/Mother-level } \\ \text { Variance (SE) }\end{array} & 0.20(0.10)^{*} & 0.15(0.08)^{*} & 0.12(0.09) & 0.09(0.04)^{*} & 0.07(0.04) & 0.05(0.02) & 0.08(0.06) \\ \begin{array}{l}\text { Explained variation } \\ \text { (PCV) \% }\end{array} & \text { Reference } & 25.0 & 40.0 & 55.0 & 65.0 & 75.0 & 60.0 \\ \begin{array}{l}\text { Log likelihood } \\ \text { Model fit statistics }\end{array} & -2486.9 & -2471.8 & -2266.1 & -2070.2 & -2436.5 & -879.4 & -941.6 \\ \text { AIC } & 4979.8 & 4959.5 & 4564.2 & 4198.4 & 4921.1 & 1860.8 & 1933.2\end{array}$

Abbreviation:- HR: Hazard ratio, SE: standard error, VPC: variance partition coefficient, ICC: intra-class correlation coefficient, PCV: proportional change in variance, AIC: Akaike information criterion, ${ }^{*} \mathrm{p}<0.05$.

\section{Fixed effects}

According to the results from 2003 NDHS presented in Model I (Table 2), inclusion of region of residence as the only covariate in the multilevel anal$y$ sis indicates that the risks of death before age five were significantly higher for children in the Northcentral (HR: I.45, $\mathrm{p}<0.05)$, North-east (HR: 2.09, $p<0.05)$, North-west (HR: 2.00, $p<0.05)$, Southeast (HR: I.36, $p<0.05)$, and South-south (HR:I.68, $\mathrm{p}<0.05)$ compared to children in the South-west. Analysis of 2008 NDHS also indicated elevated hazards of under-five death in all regions compared to South-west (in the reference category).

Results from analysis of both surveys indicated that inclusion of child-level variables into the multilevel models (Model 2, Tables 2 and 3 ) did not significantly alter the results obtained in Model I. This suggests that the effect of region where children were born or raised (on their survival chances) was independent of child-level characteristics.

In contrast, incorporating the mother-level variables into the multilevel analysis in Model 3 (Tables 2 and 3) indicated mother's attributes as important characteristics, as the risks of dying before age five became statistically insignificant in many regions. This suggests that mother-level variables included in the multilevel analysis; such as maternal education, wealth index, contraceptive use, number of children ever born and maternal age are important factors that influence variations in the risks of under-five mortality in various regions of the country. For instance, Table 2 showed that being a child of an educated woman with at least secondary education (HR: 0.70, $p<0.05$ ); being a child of a woman from households in the richest wealth quintile (HR: 0.64 , $\mathrm{p}<0.05$ ); and being a child of woman using modern contraceptives (HR: 0.67, $p<0.05$ ) was associated with lower risks of under-five mortality, irrespective of the region of residence where a child was born. Further, Model 4 considered only community-level factors in the multilevel models. Results in Model 4 Table 2 indicated that the risks of dying before age five were statistically insignificant across all regions, while results in Model 4 Table 3 indicated further reductions in the risks of dying during childhood across regions. The results in Table 3 (Model 4) suggests that the characteristics of the neighbourhood contexts tend to mitigate the risks of under-five death in some regions, while neighbourhood characteristics tend to increase risks of under-five death in the North-east (HR:I.42, $\mathrm{p}<0.05)$ and South-south (HR: I.30, p<0.05).

Results from Model 5 (full model) which incorporated all covariates as presented in Tables 2 and 3 indicated some elevated risks of under-five mortality during the period under study in the North-central (HR increased from 0.92 to I.39, $\mathrm{p}<0.05$ ); Northeast (HR increased from I.24 to I.45, p<0.05) and North-west (HR increased from I.22 to I.44, $p<0.05)$, compared to the South-west.

Further results from the full model (Model 5 Table 2) showed that child-level variables (such as child's sex, birth interval, and child's size at birth), mother-level variables (including number of CEB and contraceptive use) were significantly associated with under-five mortality. Results from 2008 dataset (Model 5 Table 3) indicate individual level factors (such as child's sex, birth interval, contraceptive use, family structure); and neighbourhood factors (such as region of residence, ethnic diversity, and community hospital delivery) as important determinants of under-five mortality in Nigeria.

Results from final model (Model 6 Table 3) showed that the risks of dying before age five were significantly higher for children in the North-central (HR: I.43, $p<0.05)$, North-east (HR: I.56, $p<0.05)$, North-west (HR: I.55, $\mathrm{p}<0.05)$ and rural areas $(H R$ : I.25, $p<0.05$ ). Model 6 also shows that residence in communities where high proportion had access to electricity (HR: 0.64, $\mathrm{p}<0.05$ - Table 2); residence in ethnically heterogeneous communities (HR: 0.82, $\mathrm{p}<0.05$ - Table 3) was significantly associated with lower risks of under-five mortality. In addition, Model 6 (Table 2) indicates that residence in poor neighbourhoods (HR: I.54, $\mathrm{p}<0.05-$ Table 2$)$, and residence in rural communities (HR: $1.25, \mathrm{p}<0.05)$ 
was associated with significantly higher risks of under-five mortality.

Finally, the decreasing values of AIC (Tables 2 and 3) with each successive model indicated a good fit of the multilevel models with each successive model signifying a significant improvement of the previous model.

Table 3 Child and mother-level compositional and community contextual factors associated with regional variations in under-five mortality in Nigeria, 2008

\begin{tabular}{|c|c|c|c|c|c|c|c|}
\hline Characteristics & Model 0 & Model I & Model 2 & Model 3 & Model 4 & Model 5 & Model 6 \\
\hline Fixed effects & $\begin{array}{l}\text { Empty Model } \\
(\mathrm{HR})\end{array}$ & $\begin{array}{l}\text { Region covariate } \\
(\mathrm{HR})\end{array}$ & $\begin{array}{l}\text { Child-level } \\
\text { variables } \\
(\mathrm{HR})\end{array}$ & $\begin{array}{l}\text { Mother-level } \\
\text { variables } \\
(\mathrm{HR})\end{array}$ & $\begin{array}{l}\text { Community- } \\
\text { level variables } \\
(\mathrm{HR})\end{array}$ & $\begin{array}{l}\text { Full model } \\
(\mathrm{HR})\end{array}$ & $\begin{array}{l}\text { Final Mode } \\
(\mathrm{HR})\end{array}$ \\
\hline \multicolumn{8}{|l|}{ Region of residence } \\
\hline South-west & & I & 1 & 1 & 1 & I & 1 \\
\hline North-central & & I.49* & $1.42 *$ & $1.33 *$ & $1.21 *$ & I.39* & $\mathrm{I} .43^{*}$ \\
\hline North-east & & $1.85 *$ & $1.73^{*}$ & $\mathrm{I} .4 \mathrm{I}^{*}$ & $\mathrm{I} .42 *$ & $\mathrm{I} .45 *$ & $1.56 *$ \\
\hline North-west & & $1.90 *$ & $1.80 *$ & $\mathrm{I} .57^{*}$ & $\mathrm{I} .44^{*}$ & $\mathrm{I} .44 *$ & $1.55^{*}$ \\
\hline South-east & & $1.6 I^{*}$ & $1.52 *$ & $1.63^{*}$ & I.59* & 1.28 & 1.36 \\
\hline South-south & & I.38* & I.40* & 1.29 & 1.30* & 1.30 & 1.31 \\
\hline \multicolumn{8}{|l|}{ Child's sex } \\
\hline Male & & & 1 & & & 1 & I \\
\hline Female & & & $0.88^{*}$ & & & $0.78^{*}$ & $0.76^{*}$ \\
\hline \multicolumn{8}{|l|}{ Birth order } \\
\hline First birth & & & 1 & & & 1 & 1 \\
\hline $2-4$ & & & $0.73^{*}$ & & & 0.95 & 0.96 \\
\hline $5+$ & & & 0.95 & & & 1.20 & 1.24 \\
\hline \multicolumn{8}{|l|}{ Birth interval } \\
\hline$<24$ months & & & 1 & & & I & 1 \\
\hline $24+$ & & & $0.54 *$ & & & $0.55 *$ & $0.55 *$ \\
\hline \multicolumn{8}{|l|}{ Child size at birth } \\
\hline Large & & & 1 & & & 1 & 1 \\
\hline Average & & & $1.22 *$ & & & $\mathrm{I} .07$ & 1.07 \\
\hline Small & & & I.46* & & & $|.3| *$ & $1.32 *$ \\
\hline \multicolumn{8}{|l|}{ Maternal education } \\
\hline None & & & & 1 & & 1 & \\
\hline Primary & & & & 0.96 & & 0.95 & \\
\hline Secondary or higher & & & & 0.98 & & 0.96 & \\
\hline \multicolumn{8}{|l|}{ Mother's age } \\
\hline $15-24$ & & & & 1 & & 1 & \\
\hline $25-34$ & & & & $0.77^{*}$ & & $0.77^{*}$ & \\
\hline $35+$ & & & & 1.01 & & 1.01 & \\
\hline \multicolumn{8}{|l|}{ CEB } \\
\hline$<3$ & & & & I & & I & 1 \\
\hline $3-4$ & & & & $1.98 *$ & & $1.76 *$ & I.76* \\
\hline $5+$ & & & & $2.37^{*}$ & & $2.34 *$ & $2.32 *$ \\
\hline \multicolumn{8}{|l|}{ Family structure } \\
\hline Monogamous & & & & 1 & & 1 & 1 \\
\hline Polygynous & & & & I.20* & & $1.15 *$ & $1.15^{*}$ \\
\hline \multicolumn{8}{|l|}{ Religion } \\
\hline Christianity & & & & 1 & & 1 & 1 \\
\hline Islam & & & & 0.85 & & 0.82 & $0.81 *$ \\
\hline Others & & & & 0.90 & & 0.95 & 0.93 \\
\hline
\end{tabular}


African Population Studies Vol 27, 2 Supp (Mar 20I4)

\begin{tabular}{|c|c|c|c|c|}
\hline \multicolumn{5}{|l|}{ Wealth index } \\
\hline Poorest & \multicolumn{2}{|l|}{ I } & \multicolumn{2}{|l|}{1} \\
\hline Poorer & \multicolumn{2}{|l|}{0.98} & \multicolumn{2}{|l|}{0.97} \\
\hline Middle & \multicolumn{2}{|l|}{1.03} & \multicolumn{2}{|l|}{$\mathrm{I} .04$} \\
\hline Richer & \multicolumn{2}{|l|}{0.99} & \multicolumn{2}{|l|}{1.06} \\
\hline Richest & \multicolumn{2}{|l|}{$0.73^{*}$} & \multicolumn{2}{|l|}{0.82} \\
\hline \multicolumn{5}{|l|}{ Contraceptive use } \\
\hline No method & \multicolumn{2}{|l|}{ I } & \multicolumn{2}{|l|}{ I } \\
\hline Traditional & \multicolumn{2}{|l|}{$0.53^{*}$} & \multicolumn{2}{|l|}{$0.53^{*}$} \\
\hline Modern & \multicolumn{2}{|l|}{$0.42 *$} & \multicolumn{2}{|l|}{$0.43^{*}$} \\
\hline \multicolumn{5}{|l|}{ Had Prenatal } \\
\hline No & \multicolumn{2}{|l|}{ I } & \multicolumn{2}{|l|}{ I } \\
\hline Yes & \multicolumn{2}{|l|}{$0.85^{*}$} & $0.84 *$ & \\
\hline Place of delivery & & & & \\
\hline Home & I & & I & \\
\hline Health facility & 1.21 & & 1.27 & \\
\hline Place of residence & & & & \\
\hline Urban & & I & I & 1 \\
\hline Rural & & $1.19 *$ & 1.17 & $1.25 *$ \\
\hline Ethnic diversity & & & & \\
\hline Homogenous & & I & I & I \\
\hline Mixed & & 0.98 & $0.81 *$ & $0.82 *$ \\
\hline Heterogeneous & & 1.01 & $0.74 *$ & $0.82 *$ \\
\hline Community mater & & & & \\
\hline Low & & 1 & I & \\
\hline Medium & & 0.96 & 1.03 & \\
\hline High & & 0.94 & I.II & \\
\hline Community prenat & & & & \\
\hline Low & & I & I & \\
\hline Medium & & 1.11 & 1.11 & \\
\hline High & & I.II & 1.20 & \\
\hline Proportion with ele & & & & \\
\hline Low & & I & I & \\
\hline Medium & & 1.04 & 1.04 & \\
\hline High & & 0.95 & 0.97 & \\
\hline Proportion with pi & & & & \\
\hline Low & & I & I & \\
\hline Medium & & 0.88 & 0.85 & \\
\hline High & & $0.85 *$ & 0.88 & \\
\hline Community hospit & & & & \\
\hline Low & & I & 1 & \\
\hline Medium & & $0.87^{*}$ & 0.88 & \\
\hline High & & $0.7 I^{*}$ & $0.77 *$ & \\
\hline Community povert & & & & \\
\hline Low & & I & 1 & \\
\hline Medium & & 1.11 & 1.01 & \\
\hline High & & $1.23 *$ & 1.07 & \\
\hline Distance to health & & & & \\
\hline Big problem & & I & I & \\
\hline Not a big problem & & 1.02 & 1.10 & \\
\hline
\end{tabular}




\begin{tabular}{|c|c|c|c|c|c|c|c|}
\hline Random effects & Empty & Region & Child & Mother & Community & Full model & Final model \\
\hline \multicolumn{8}{|l|}{ Community-level } \\
\hline Variance (SE) & $0.21(0.09)^{*}$ & $0.14(0.06)^{*}$ & $0.14(0.07)$ & $0.12(0.05)^{*}$ & $0.09(0.05)^{*}$ & $0.07(0.04)$ & $0.04(0.02)^{*}$ \\
\hline VPC/ICC (\%) & 6.0 & 4.1 & 4.1 & 3.5 & 2.7 & 2.1 & 1.2 \\
\hline $\begin{array}{l}\text { Explained variation } \\
\text { (PCV) \% }\end{array}$ & Reference & 33.3 & 33.3 & 42.9 & 57.1 & 66.7 & 81.0 \\
\hline \multicolumn{8}{|l|}{ Child/Mother-level } \\
\hline Variance (SE) & $0.14(0.08)^{*}$ & $0.12(0.06)^{*}$ & $0.11(0.09)$ & $0.09(0.07)$ & $0.06(0.03)$ & $0.04(0.03)$ & $0.04(0.02)$ \\
\hline $\begin{array}{l}\text { Explained variation } \\
\text { (PCV) \% }\end{array}$ & Reference & 14.3 & 21.4 & 35.7 & 57.1 & 71.4 & 71.4 \\
\hline Log likelihood & -10179.8 & -10139 & -10004 & -1433.5 & -10090.8 & $-4|2|$ & -3870.2 \\
\hline \multicolumn{8}{|l|}{ Model fit statistics } \\
\hline $\mathrm{AIC}$ & 20365.5 & 20294.0 & 20032.1 & 8327.0 & 20229.5 & 8334.4 & 7792.3 \\
\hline
\end{tabular}

Abbreviation:- HR: Hazard ratio, SE: standard error, VPC: variance partition coefficient, , ICC: intra-class correlation coefficient, PCV: proportional change in variance, AIC: Akaike information criterion, ${ }^{*} \mathrm{p}<0.05$.

\section{Discussion}

This paper examines the effects of neighbourhood contexts on under-five mortality in Nigeria. Multilevel Cox proportional hazard model was employed to account for the hierarchical nature of the DHS data. This is because the children were nested within mothers, and mothers were nested within communities.

Findings showed the importance of both individual-level characteristics (i.e. mother and child levels) and community or neighbourhood contexts for child survival in Nigeria. For instance, results from the null model indicated a significant variation in under-five mortality across communities. This result suggests that situations of the community contexts where children are born or raised significantly influence under-five mortality in Nigeria. This underscores the need to take neighbourhood contexts into consideration (Aremu et al., 20I I; Boco, 20l0) in the efforts to address the high level of under-five mortality in the country.

Findings in this paper established a strong association between under-five mortality and region of residence. As previously found (Antai, 20ll lb), this result indicates region of residence where children were born or raised as important contextual factors influencing under-five mortality in Nigeria. In particular, the results demonstrated that while characteristics of the neighbourhood contexts appear to alleviate under-five mortality risks in the South-west Nigeria, community characteristics tend to aggravate risks of under-five mortality in other regions, especially North-east, North-west and South-south. Sastry (1996) noted that characteristics of the community contexts can either mitigate or exacerbate mortality risks of individuals depending on the neighbourhood where individuals reside.

Findings further established significant relationship between under-five mortality and other neigh- bourhood characteristics such as place of residence, community poverty, and community infrastructure. For instance, neighbourhood infrastructure such as access to electricity in a community was established as important predictor of under-five mortality in Nigeria. This result points to the importance of good infrastructural contexts for child survival. Constant electric supply could possibly help to prevent some infectious diseases through the use of electrical appliances such as fridge (to preserve foodstuffs) and microwaves (to keep food warm for children). In addition, availability and access to drinkable water within the neighbourhood could prevent children from contacting avoidable infections like waterborne diseases such as diarrheal disease and other forms of infectious diseases. Sastry (1996) had showed that infrastructures such as electricity and water supply are significantly related to child survival.

The socio-economic context of the community where children live was also found as important predictor of under-five mortality in the country. It was shown that poverty concentration within a community appears to significantly increase risks of underfive mortality. Factors that contribute to poor socioeconomic contexts in Nigeria include poor infrastructure (National Planning Commission, 2004). Nigeria is one of the sub-Saharan African countries that face most critical infrastructural challenge (Akinwale, 2010; Foster \& Pushak, 20II). The negative effect of poor socio-economic context on child survival is expected to be severe in a country like Nigeria where there is a high level of infrastructural deficiency.

Corrupt practices at various levels of governance in Nigeria have led to non-availability of good infrastructures in the country (National Planning Commission, 2004; Osoba, 1996). Since successive Nigeria governments have failed in their roles to 
provide social services such as good roads, electricity, piped-borne water, health facilities as well as adequate securities among others, the usual practice now is for the relatively better-off communities to make communal arrangements to provide services such as bore-holes, dispensaries, motorable roads and even vigilante groups for their communal benefits. While these activities may be affordable for very rich and relatively better-off communities, communities predominantly occupied by poor people would find it difficult to make provision for such services. Thus, the present study established an elevated risk of under-five mortality for communities that had poor socio-economic contexts, perhaps because young children are likely to be highly vulnerable to unfavourable community contexts arising from lack of essential social services. This establishes one of the reasons why poor neighbourhoods like slums tend to have poor child health outcomes (Unger, 2013).

Results of this study provide empirical explanations supporting the adopted theories which posit that community or neighbourhood contexts matter for health outcomes of individuals. Findings of this study suggest that achieving improved neighbourhood contexts holds great potentials for acceleration of under-five mortality reduction in Nigeria.

\section{Conclusion}

This study found that substantial variations in underfive mortality exist across neighbourhood and regions in Nigeria, with economically and socially deprived neighbourhoods and regions having elevated risks of under-five mortality compared to better-off communities and regions. Findings of this study suggest that efforts to attain Millennium Development Goal four (childhood mortality reduction) should not only include policies that address individual-level factors. Government and well-meaning individuals are urgently needed to intensify efforts towards undertaking neighbourhood or communitylevel interventions aimed at improving child survival in the socially and economically deprived neighbourhoods and regions.

\section{Limitations}

The following limitations should be borne in mind while interpreting the findings of this study. First, the study adopted PSU or cluster as a proxy for neighbourhood. This may bring about information biases as a result of misclassifying respondents into wrong administrative boundaries (Antai, 20lla). Second, some important community-level factors such as measurable distance to health facility and those related to cultural practices and norms were not available and could not be considered in our analysis. Third, aggregating the individual-level variables at PSU level could lead to multicollinearity. This was however minimized by correlation test and fitting of stepwise regression. It is however recommended that future investigations should consider use of surveys and censuses that collect community-level variables.

\section{Notes}

I. The following terms are used interchangeably in this paper:

"Community characteristics" and "neighbourhood characteristics".

"Community or neighbouhood infrastructure" and "community electric connection and piped water";

"community socio-economic contexts" and "community poverty"

2. Community-level variables are categorized as low, medium and high; where low refers to the lowest tertile, medium is the middle tertile, and high refers to the upper tertile.

3. The intra-class correlation (ICC) is an important measure of the relatedness of clustered data within community or household units (Antai 20II). ICC is the proportion of the total variance that is at the community (Pillinger, 20l I). The VPC was calculated in this study using the linear threshold model method whereby VPC corresponds to the intra-class correlation (ICC) (Merlo et al., 2006).

Hence, the VPC was computed using:

$\rho=\left(\delta^{2} \mu /\left(\delta^{2} \mu+\left(\pi^{2} / 3\right)\right)\right.$

Where, $\rho \rho$ is the ICC, $\delta^{2} \mu \delta^{2} \mu$ is the variance at the community level, $\pi^{2} / 3 \pi^{2} / 3=3.29$ and represents the fixed variance at individual level (Merlo et al., 2006).

\section{Acknowledgements}

The research leading to these results has received funding from a fellowship award provided by the Consortium for Advanced Research Training in Africa (CARTA). CARTA has been funded by the Wellcome Trust (UK) (grant: 087547/Z/08/Z), the Department for International Development under the Development Partnerships in Higher Education (DelPHE), the Carnegie Corporation of New York (grant: B 8606), the Ford Foundation (grant:II00-0399), and the Bill and Melinda Gates Foundation (grant: 5I228). Funding from the Fogarty Foundation supporting my Postdoctoral Fellowship at Wits School of Public Health is gratefully acknowledged. An ear- 
lier version of this paper was presented at the $27^{\text {th }}$ International Population Conference of the International Union for the Scientific Study of Population (IUSSP) held in Busan, South Korea, from 26-3 I September, 2013. Insightful comments from conference participants are gratefully acknowledged. Author is also grateful to ICF Macro for permission to use Nigeria DHS data.

\section{References}

Adebowale, Ayo, Yusuf, Bidemi, \& Fagbamigbe, Adeniyi. (20I2). Survival probability and predictors for woman experience childhood death in Nigeria: "analysis of north-south differentials". BMC Public Health, I2(I), 430.

Akinbami, F.O, Hamzat T.H, Orimadegun A.E, Tongo O, Oyeyemi L, Okafor O, \& Akinyinka O.O. (2010). Body mass composition: a predictor of admission outcomes among hospitalized Nigerian under 5 children. Asia Pac Journal of Clinical Nutrition, 19(3).

Akinwale, Akeem Ayofe. (2010). The Menace of Inadequate Infrastructure in Nigeria. African Journal of Science, Technology, Innovation and Development, 2(3), 207-228.

Aminzadeh, K., Denny, S., Utter, J., Milfont, T. L., Ameratunga, S., Teevale, T., \& Clark, T. (20I3). Neighbourhood social capital and adolescent selfreported wellbeing in New Zealand: a multilevel analysis. Soc Sci Med, 84, I3-21. doi: 10.1016/ j.socscimed.2013.02.012

Antai, Diddy. (20I la). Inequalities in under-5 mortality in Nigeria: do ethnicity and socioeconomic position matter? J Epidemiology, 2I(I), I3-20.

Antai, Diddy. (20I Ib). Regional inequalities in under5 mortality in Nigeria: a population-based analysis of individual- and community-level determinants. Population Health Metrics, 9(6), I-27.

Aremu, Olatunde, Lawoko, Stephen, Moradi, Tahereh, \& Dalal, Koustuv. (20II). Socioeconomic determinants in selecting childhood diarrhoea treatment options in Sub-Saharan Africa: A multilevel model. Italian journal of paediatrics 37(13), 2-8.

Babalola, Stella, \& Fatusi, Adesegun. (2009). Determinants of use of maternal health services in Nigeria - looking beyond individual and household factors. BMC Pregnancy and Childbirth, 9(I), 43.

Becares, L., Cormack, D., \& Harris, R. (20I3). Ethnic density and area deprivation: neighbourhood effects on Maori health and racial discrimination in Aotearoa/New Zealand. Soc Sci Med, 88, 7682. doi: 10.1016/j.socscimed.2013.04.007
Becher, H. (20I0). Analysis of Mortality Clustering at member HDSSs within the INDEPTH Network an important public health issue. Global Health action Supplement I, 3-7.

Boco, Adébiyi Germain. (2010). Individual and Community Level Effects on Child Mortality: An Analysis of 28 Demographic and Health Surveys in Sub-Saharan Africa. In DHS Working Papers No. 73 (Ed.). Calverton, Maryland, USA: ICF Macro.

Ekenze, S.O., Anyanwu K.K, \& Chukwumam D.O. (2009). Childhood trauma in Owerri (south eastern) Nigeria. Nigeria Journal of Medicine, I8(I), 79-83.

Fayeun, Olufunke, \& Omololu, Obafemi. (20I I). Ethnicity and Child Survival in Nigeria. African Population Studies, 25 Supplement(I), 92-I I 2.

Foster, Vivien, \& Pushak, Nataliya. (20I I). Nigeria's Infrastructure: A Continental Perspective. Africa Infrastructure Country Diagnostic. Washington DC, USA: The World Bank.

Fox, John. (2002). Cox Proportional-Hazards Regression for Survival Data. Appendix to An R and S-PLUS Companion to Applied Regression.

Gilbert, N. L., Auger, N., Wilkins, R., \& Kramer, M. S. (20I3). Neighbourhood Income and Neonatal, Postneonatal and Sudden Infant Death Syndrome (SIDS) Mortality in Canada, I99I-2005. Can J Public Health, 104(3), el87-192.

Grais, R.F., Dubray C, Gerstl S, Guthmann J.P, Djibo A, Nargaye K.D, . . Guerin P.J. (2007). Unacceptably high mortality related to measles epidemics in Niger, Nigeria, and Chad. PLoS Med, 4(I).

Griffiths, P., Madise N, Whitworth A, \& Mathew Z. (2004). A tale of two continents: a multilevel comparison of the determinants of child nutritional status from selected African and Indian regions. Health \& Place, 10, 183-199.

Harttgen, K., \& Misselhorn, M. (2006). A Multilevel Approach to Explain Child Mortality and Undernutrition in South Asia and Sub-Saharan. Ibero-America Institute for Economic Research, $\mathrm{Nr}$ 156(I-38).

Ifesanya, A.O, Afuwape D, Okoje V.N, Agunloye A, Odole O, Okolo C.A, \& Alonge T.O. (2009). Unintentional injury outcomes secondary to pedestrian traffic crashes: a descriptive analysis from a major medical center. Prehosp Disaster Med, 24(5), 443-446.

Lykens, Kristine, Karan P Singh, \& Elewichi Ndukwe. (2009). Social, Economic, and Political Factors in Progress Towards Improving Child Survival in Developing Nations. Journal of Health Care for the Poor and Underserved, 20(4), I37-I 48.

Melliez, H, Boelle P.Y, Baron S, Mouton Y, \& 
Yazdanpanah Y. (2007). Effectiveness of childhood vaccination against rotavirus in subSaharan Africa: the case of Nigeria. Vaccine, 25(2), 298-305.

National Planning Commission. (2004). National Economic Empowerment and Development Strategy (NEEDS). Abuja, Nigeria.

NPC \& ICF Macro. (2009). Nigeria 2008 Demographic and Health Survey.

Nwokocha, E.E, \& Awomoyi, A.O. (2009). Factors influencing mothers' role in convulsion treatment among under-five children in Ibadan, Nigeria. World Health Popul. 2009; I I (2): I5-29., I I(2), I529.

Okafor, U.V, Efetie E.R, lgwe W, \& Okezie O. (2009). Anaesthetic management of patients with preeclampsia/eclampsia and perinatal outcome. Journal of Matern Fetal Neonatal Med, 22(8), 688692.

Okoro, P.E, lgwe P.O, \& Ukachukwu A.K. (2009). Childhood burns in south eastern Nigeria. Africa Journal Paediatric Surgery, 6(I), 24-27.

Omariba, D. Walter Rasugu, Roderic Beaujot, \& Fernando Rajulton. (2007). Determinants of infant and child mortality in Kenya: an analysis controlling for frailty effects. Popul Res Policy Rev, 26, 299-321.

Osoba, S. O. (1996). Corruption in Nigeria: historical perspectives. Review of African Political Economy, 23(69), 37I-386. doi: I0.1080/ 03056249608704203
Pillinger, Rebecca. (20II). A transcript of measuring dependency presentation. Centre for Multilevel Modelling, University of Bristol

Rabe-Hesketh, Sophia, Skrondal, Anders, \& Picklesz, Andrew. (2004). GLLAMM Manual, University of California, Berkeley, Division of Biostatistics Working Paper Series,

Reviews, Book. (1988). Book Reviews. Journal of the American Statistical Association, 83(403), 902-926. doi: 10.1080/0162I459.1988.10478680

Sastry, Narayan. (1996). Community Characteristics, Individual and Household Attributes, and Child Survival in Brazil. Demography, 33(2), 2I I-229.

Sastry, Narayan. (1997). Family-level clustering of childhood mortality risk in Northeast Brazil. Population Studies, 5I, 245-26I.

Unger, A. (2013). Children's health in slum settings. Arch Dis Child. doi: 10.1 136/archdischild-20II301621

WHO. (2003). An analytical framework for the study of child survival in developing countries by Mosley W.H and Chen L.C. Bulletin of the World Health Organization, 8I(2), I40-I45.

World Factbook. (20II, Accessed on February 28, 20 I I.). Fact book, from http://en.wikipedia.org/ wiki/The_World_Factbook

Zanini, R R, B, Moraes A, J, Giugliani E R, \& J, Riboldi. (2009). Infant mortality trends in the State of Rio Grande do Sul, Brazil, 1994-2004: a multilevl analysis and community risk factors. Cad. Saude Publica, Rio de Janerio, 25(5), I035- 1045. 